\title{
Predicting non-sentinel node metastasis during surgery in breast cancer patients with one to three positive sentinel node(s) on a frozen biopsy result after neoadjuvant chemotherapy
}

Jung Whan Chun ( $\sim$ junghwanny@naver.com )

Asan Medical Center https://orcid.org/0000-0001-6438-5526

Jisun Kim

Asan Medical Center

II Yong Chung

Asan Medical Center

Beom Seok Ko

Asan Medical Center

Hee Jeong Kim

Asan Medical Center

Jong Won Lee

Asan Medical Center

Byung Ho Son

Asan Medical Center

Sei-Hyun Ahn

Asan Medical Center

Sae Byul Lee

Asan Medical Center https://orcid.org/0000-0002-3370-6937

\section{Research Article}

Keywords: Breast cancer, Sentinel lymph node, Non-sentinel lymph node, Nomogram

Posted Date: February 2nd, 2022

DOI: https://doi.org/10.21203/rs.3.rs-1257765/v1

License: (c) (i) This work is licensed under a Creative Commons Attribution 4.0 International License.

Read Full License 


\section{Abstract}

\section{Purpose}

Our goal was to develop a tool that could accurately predict the possibility of non-sentinel lymph node metastasis (NSLNM) during surgery, allowing a surgeon to decide the extent of further axillary lymph node dissection (ALND) intraoperatively for patients with one to three positive sentinel lymph node(s) (SLN) after neoadjuvant chemotherapy (NAC).

\section{Methods}

In a retrospective analysis of the Asan Medical Center (AMC) database, we included 558 patients' records who were treated between 2005 and 2019. Using chi-square and logistic regression with a bootstrapped, backward elimination method, 13 factors were assessed for their utility in predicting NSLNM. Based on the results of the univariate analysis for statistical significance, the number of positive SLN(s), number of frozen nodes, progesterone receptor (PR) positivity and clinical $\mathrm{N}$ stage were selected for the multivariate analysis and used to generate a nomogram for predicting residual nodal disease. The resulting nomogram was validated using a more recent, different time window patient group at the AMC.

\section{Results}

We designed a nomogram to predict NSLNM that included four components: number of SLN(s), number of frozen nodes, PR positivity and clinical $\mathrm{N}$ stage prior to NAC. The area under the receiver operating characteristics curve value of this formula was $0.709(95 \% \mathrm{Cl}, 0.658-0.761)$ for the development set and $0.715(95 \% \mathrm{Cl}, 0.634-0.796)$ for the validation set.

\section{Conclusion}

This newly developed AMC nomogram may be useful to a surgeon for intraoperative guidance in determining the extent of further axillary surgery.

\section{Introduction}

Although previous trials of the survival benefit for patients who underwent neoadjuvant chemotherapy (NAC) failed to demonstrate a relative superiority over patients treated with adjuvant chemotherapy, the addition of NAC for treatment of eligible breast cancer patients has been widely accepted. NAC can help reduce the need for total mastectomy, full axillary lymph node dissection (ALND) and the associated morbidity without increasing locoregional recurrence [1]. Sentinel lymph node biopsy (SLNB) after NAC in patients with clinically positive axilla resulted in acceptable accuracy, establishing it as a viable axillary management strategy [1-3]. Our institutional practice pattern has also included SLNB as the initial approach for the axilla after NAC unless the patient had a significant disease burden remained or progressive disease. 
According to the recent National Comprehensive Cancer Network (NCCN) guidelines, complete ALND has been the standard surgical management for patients who presented with node-positive breast cancer after NAC [4]. Non-sentinel lymph nodes (NSLNs) were found to be tumour free in a significant proportion of breast cancer patients with positive SLNs who had received ALND. Among 160 patients with macrometastasis to SLNs, Dingemans et al. reported that $59 \%$ of primary breast cancer patients lacked NSLNM. These patients might received unnecessary ALND, which could have resulted in significant complications and provided no therapeutic benefits [5]. Jeruss et al. conducted a study that included 104 patients who received NAC, had a positive SLN and underwent ALND between 1997 and 2005 [6]. Of their research cohort, $44 \%$ did not have positive non-SLNs. They looked at factors such as lymphovascular invasion (LVI), method of SLN metastasis detection, multicentricity, ALN status at presentation and pathological tumour size to see if they could predict additional NSLNM. Based on this finding, they derived the MD Anderson nomogram, which has a significant AUC. In addition, 132 patients were followed prospectively between 2001 and 2007 in a study by Gimbergues et al. All patients were given NAC and had SLN biopsy with ALND levels I and II [7]. They reported that $47.1 \%$ of their patient population did not have NSLNM, and they tested the accuracy of previous nomograms from the Memorial Sloan-Kettering Cancer Center, the MD Anderson Cancer Center and the Tenon Hospital in Paris, with AUC values ranging from 0.7 to 0.8 .

However, these nomograms are mostly based on factors from a final pathology report after surgery, such as LVI, pathologic tumour size and the size of SLN metastasis, among others. Thus, when indicated, patients are expected to undergo further axillary dissection on a separate schedule. In this study, we retrospectively analysed patient data from the Asan Medical Center (AMC) to develop a nomogram that could help predict the possibility of NSLNM based on the clinical information available before a planned surgery.

\section{Material And Methods}

\section{Patients}

We reviewed data from patients who had breast surgery with ALND after NAC between 2005 and 2019 . Patients with one to three metastasis-positive sentinel node(s) treated with standard axillary procedure were included in the study. According to the oncologists at the AMC, all included patients received a full course of standard neoadjuvant therapy. As a result of ALND, we could identify patients with or without residual nodal disease based on the final pathology reports. We excluded patients who had bilateral breast cancer, inflammatory breast cancer or had distant metastasis at the time of presentation, as well as those who had more than four sentinel nodes positive for metastasis and went straight to ALND without SNB or SNB only. We also excluded patients whose NAC was incomplete due to intolerance or refusal. Finally, 558 patients were chosen for further analysis. The records of 384 patients treated between 2005 and 2016 were utilized to develop the prediction model, and the data of 174 patients treated from 2017 to 2019 were used to validate the generated prediction model. 
The data of the patients were reviewed for the total number of metastatic nodes on the final pathology report, intraoperative frozen section biopsy result of sentinel node(s), presence or absence of additional metastatic non-sentinel nodes and number, tumour invasion depth, tumour biology, initial clinical stage before chemotherapy and radiology report of ultrasonogram or MRI of breast before and after chemotherapy.

This study was approved by the Institutional Review Board of the Asan Medical Center, Seoul, South Korea (20171341). Because the study was based on retrospective clinical data, informed consent was not required.

\section{Preoperative chemotherapy and SNLB mapping method}

NAC was administered to a patient every 3 weeks, and a regimen was selected from among standard proposed regimens based on the clinical stage or tumour biology of a patient. Although standard regimens are constantly evolving, the oncologists at our institution generally followed the most recent NCCN guideline of the time. The surgery was performed 3-4 weeks after the completion of preoperative chemotherapy. We assessed a patient's response to NAC using either an ultrasonogram or an MRI of the breast before and after treatment. According to the Revised Response Evaluation Criteria in Solid Tumors (RECIST guideline, version 1.1), we defined partial remission as a decrease of more than $30 \%$ in the sum of the longest diameters of the target lesions compared with baseline. In addition, we used the term complete remission when we discovered the disappearance of all target lesions. All tumours that did not meet the above criteria were classified as stable disease.

We used ${ }^{99 m}$ Tc-sulphur colloid diluted in normal saline as a radiopharmaceutical agent with gamma probe detection (NeoProbe2000, US surgical, Norwalk, CT) for SLN identification. We injected the mapping agent periareolarly and massaged the breast for 5 minutes. Along with the most radioactive nodes, clinically enlarged, firm or palpable axillary lymph nodes without an active gamma signal were excised and counted as part of the total number of SLNs.

\section{Statistical analysis}

To identify significant factors that predict the possibility of residual disease in non-sentinel nodes, we first included the following parameters in the univariate analysis: age at diagnosis, tumour grade, hormone receptor score, HER2 status, classification into four subtypes (HR+/HER2-, HR+/HER2+, HR-HER2+, HR-/HER2-), Ki-67, clinical T stage and N stage before NAC and its degree of response to therapy, number of metastatic sentinel nodes, total number of submitted sentinel nodes for frozen section biopsy and the greatest tumour invasion depth of the sentinel nodes.

We used the data from 384 patients treated between 2005 and 2016 for the development of the prediction model and the data from 174 patients treated from 2017 to 2019 for validation. In the development set, univariate assessment of these factors was performed using a logistic regression model. A multivariable logistic regression model was used to further analyse and generate a prediction model for the possibility of residual nodal disease after NAC when one to three sentinel nodes were positive intraoperatively. The 
predictors for the multivariable model were selected using backward elimination in more than half of the 1000 bootstrap resamples. The final model was estimated using penalised maximum likelihood and was presented as a nomogram. The discrimination ability of the nomogram was assessed by using area under the receiver operating characteristic curve (AUC). The calibration ability was assessed using the calibration plot and the Hosmer-Lemeshow test. We performed an internal validation with bootstrapping with 1000 iterations, calculating optimism-corrected AUC (C statistics). In validation set, the discrimination and calibration abilities were also evaluated. All tests were two-sided, and p-value of less than 0.05 was considered statistically significant. Statistical analysis was conducted with SPSS statistics version 23.0 (IBM Corp., Armonk, USA) and R (version 3.6.1; R Foundation for Statistical Computing, https://www.Rproject.org).

\section{Results}

\section{Baseline characteristics}

Table 1 shows the demographics of the 558 patients whose clinical data was used to develop the nomogram. In total, the majority of patients were under the age of $50(63.6 \%)$, had a single SLN metastasis at the time of surgery $(50.9 \%)$, had three to five frozen biopsy sent for pathologic confirmation of SLN status (66.1\%), were in clinical T stage $2(63.4 \%)$. Furthermore, a substantial portion of our patients had N1 stage disease both before $(74.4 \%)$ and after $(75.1 \%)$ NAC. The majority of tumours were low grade (81\%), oestrogen receptor positive (80.6\%), progesterone receptor positive (66.5\%), HER2 negative (78.3\%), biological subtype of hormone receptor positive and HER2 negative (78.3\%). As shown in the Table 1, the baseline characteristics of patients with or without residual nodal disease differed significantly. The residual nodal disease group had more oestrogen receptor-positive patients $(85.7 \%, p=0.007)$ and more progesterone receptor-positive patients $(72.2 \%, p=0.011)$ than the no nodal residual disease group. In addition, patients with nodal residual disease had a higher initial $\mathrm{N}$ stage $(\mathrm{p}<0.001)$, a higher number of positive SLN $(p<0.001)$, a higher pathologic T stage $(p=0.001)$ and a higher $N$ stage $(p<0.001)$ than those without nodal residual disease. 
Table 1

Baseline patient characteristics and association between residual disease and clinicopathologic variables

\begin{tabular}{|c|c|c|c|c|}
\hline & \multirow[t]{2}{*}{ Total } & \multicolumn{2}{|c|}{ Residual disease } & \multirow[t]{2}{*}{$P$ value } \\
\hline & & No & Yes & \\
\hline Number of patients & 558 & 313 & 245 & \\
\hline Age & & & & 0.035 \\
\hline$<50$ years & $355(63.6)$ & $211(67.4)$ & $144(58.8)$ & \\
\hline$\geq 50$ years & $203(36.4)$ & $102(32.6)$ & $101(41.2)$ & \\
\hline Tumour grade & & & & 0.002 \\
\hline $\mathrm{G} 1 / \mathrm{G} 2$ & $452(81.0)$ & 242 (77.3) & $210(85.7)$ & \\
\hline G3 & $102(18.3)$ & $71(22.7)$ & $31(12.7)$ & \\
\hline Unknown & $4(0.7)$ & $0(0.0)$ & $4(1.6)$ & \\
\hline Oestrogen receptor & & & & 0.007 \\
\hline Negative & $108(19.4)$ & 73 (23.3) & $35(14.3)$ & \\
\hline Positive & $450(80.6)$ & 240 (76.7) & $210(85.7)$ & \\
\hline Progesterone receptor & & & & 0.011 \\
\hline Negative & $187(33.5)$ & $119(38.0)$ & $68(27.8)$ & \\
\hline Positive & $371(66.5)$ & $194(62.0)$ & $177(72.2)$ & \\
\hline HER2 status & & & & 0.393 \\
\hline Negative & $437(78.3)$ & $241(77.0)$ & $196(80.0)$ & \\
\hline Positive & $121(21.7)$ & $72(23.0)$ & $49(20.0)$ & \\
\hline Biological subtype & & & & 0.039 \\
\hline HR+/HER2- & $379(67.9)$ & $199(63.6)$ & $180(73.5)$ & \\
\hline HR+/HER2+ & $72(12.9)$ & $41(13.1)$ & $31(12.7)$ & \\
\hline HR-/HER2+ & $44(7.9)$ & $30(9.6)$ & $14(5.7)$ & \\
\hline HR-/HER2- & $63(11.3)$ & $43(13.7)$ & $20(8.2)$ & \\
\hline Initial T stage & & & & 0.165 \\
\hline 1 & $54(9.7)$ & $32(10.2)$ & $22(9.0)$ & \\
\hline 2 & $354(63.4)$ & $208(66.5)$ & $146(59.6)$ & \\
\hline 3 & $138(24.7)$ & $66(21.1)$ & $72(29.4)$ & \\
\hline
\end{tabular}




\begin{tabular}{|c|c|c|c|c|}
\hline \multirow[b]{2}{*}{4} & \multirow{2}{*}{$\begin{array}{l}\text { Total } \\
12(2.2)\end{array}$} & \multicolumn{2}{|c|}{ Residual disease } & \multirow[t]{2}{*}{$P$ value } \\
\hline & & $7(2.2)$ & $5(2.0)$ & \\
\hline Initial N stage & & & & $<0.001$ \\
\hline 0 & $92(16.5)$ & $65(20.7)$ & $27(11.0)$ & \\
\hline 1 & $415(74.4)$ & $229(73.2)$ & $186(75.9)$ & \\
\hline 2 & $51(9.1)$ & $19(6.1)$ & $32(13.1)$ & \\
\hline Response to NAC & & & & 0.891 \\
\hline Complete remission & $22(3.9)$ & $14(4.5)$ & $8(3.3)$ & \\
\hline Partial remission & $406(72.8)$ & $228(72.8)$ & 178 (72.7) & \\
\hline Stable disease & $119(21.3)$ & 65 (20.8) & $54(22.0)$ & \\
\hline Progressive disease & $11(2.0)$ & $6(1.9)$ & $5(2.0)$ & \\
\hline Surgery type & & & & 0.079 \\
\hline Breast-conserving surgery & $258(46.2)$ & $155(49.5)$ & $103(42.0)$ & \\
\hline Mastectomy & $300(53.8)$ & $158(50.5)$ & $142(58.0)$ & \\
\hline Number of positive SLNs & & & & $<0.001$ \\
\hline 1 & $284(50.9)$ & $185(59.1)$ & $99(40.4))$ & \\
\hline 2 & $203(36.4)$ & 106 (33.9) & $97(39.6)$ & \\
\hline 3 & $71(12.7)$ & $22(7.0)$ & $49(20.0)$ & \\
\hline Pathologic T stage & & & & 0.001 \\
\hline 0 & $29(5.2)$ & $18(5.8)$ & $11(4.5)$ & \\
\hline 1 & $235(42.1)$ & 150 (47.9) & $85(34.7)$ & \\
\hline 2 & 238 (42.7) & $124(39.6)$ & $114(46.5)$ & \\
\hline 3 & $56(10.0)$ & $21(6.7)$ & $35(14.3)$ & \\
\hline Pathologic N stage & & & & $<0.001$ \\
\hline 1 & $419(75.1)$ & 309 (98.7) & $110(44.9)$ & \\
\hline 2 & 139 (24.9) & $4(1.3)$ & $135(55.1)$ & \\
\hline SLN: sentinel lymph node & & & & \\
\hline NAC: neoadjuvant chemotl & & & & \\
\hline
\end{tabular}


Response to NAC: followed the Revised Response Evaluation Criteria in Solid Tumors (RECIST) guideline, version 1.1

Table 2 shows the clinicopathologic information of patients based on their baseline and validation groups. The median age of the test population was 46.4 years (range 24-74) and 48.3 years (range 26-78) for the validation group. The hormone receptor status and HER2 positivity did not significantly differ between the two groups. The mean clinical tumour size before NAC was $44.2 \mathrm{~mm}$ (Standard Deviation (SD) $20.3 \mathrm{~mm}$ ) in the test population and $38.2 \mathrm{~mm}$ (SD $18.7 \mathrm{~mm}$ ) in the validation group. The mean pathologic tumour size after NAC was $21.4 \mathrm{~mm}$ (SD $15.9 \mathrm{~mm}$ ) for the test population $(50.1 \%$ mean reduction) and $18.7 \mathrm{~mm}$ (SD $14.2 \mathrm{~mm}$ ) (50.2\% mean reduction) for the validation group. Clinical parameters of both groups of patients exhibited no significant difference except for clinical N stage, where the proportion of N2 patients was $7.3 \%$ in the test group and $16.2 \%$ in the validation group. The number of positive SLN(s) at the time of surgery and the proportion of patients with residual nodal disease were critical parameters in developing the nomogram, and its $p$-values failed to demonstrate statistical significance. Supplementary Table 1 shows the comparison of detailed clinicopathologic factors between the baseline and validation groups based on residual nodal disease. 
Table 2

Comparison of the baseline group and validation group

\begin{tabular}{|c|c|c|c|}
\hline & Baseline group & Validation group & $P$ value \\
\hline Number of patients & 384 & 174 & \\
\hline Age at diagnosis & & & 0.203 \\
\hline$<50$ years & $251(65.4)$ & $104(59.8)$ & \\
\hline$\geq 50$ years & $133(34.6)$ & $70(40.2)$ & \\
\hline Tumour grade & & & 0.849 \\
\hline $\mathrm{G} 1 / \mathrm{G} 2$ & $313(81.5)$ & $143(82.2)$ & \\
\hline G3 & $71(18.5)$ & $31(17.8)$ & \\
\hline Oestrogen receptor & & & 0.698 \\
\hline Negative & $76(19.7)$ & $32(18.4)$ & \\
\hline Positive & $308(80.3)$ & $142(81.6)$ & \\
\hline Progesterone receptor & & & 0.475 \\
\hline Negative & $125(32.6)$ & $62(35.6)$ & \\
\hline Positive & $259(67.4)$ & $112(64.4)$ & \\
\hline HER2 status & & & 0.545 \\
\hline Negative & $298(77.6)$ & 139 (79.9) & \\
\hline Positive & $86(22.4)$ & $35(20.1)$ & \\
\hline Biological subtype & & & 0.359 \\
\hline HR+/HER2- & $260(67.7)$ & $119(68.4)$ & \\
\hline HR+/HER2+ & $49(12.8)$ & $23(13.2)$ & \\
\hline HR-/HER2+ & $35(9.1)$ & $9(5.2)$ & \\
\hline HR-/HER2- & $40(10.4)$ & $23(13.2)$ & \\
\hline Clinical T stage & & & 0.051 \\
\hline T1 & $32(8.4)$ & $22(12.6)$ & \\
\hline T2 & $245(63.8)$ & 109 (62.6) & \\
\hline T3 & $95(24.7)$ & $43(24.8)$ & \\
\hline $\mathrm{T} 4$ & $12(3.1)$ & 0 & \\
\hline Clinical N stage & & & 0.032 \\
\hline
\end{tabular}




\begin{tabular}{|c|c|c|c|}
\hline & Baseline group & Validation group & $P$ value \\
\hline No & $70(18.2)$ & $22(12.6)$ & \\
\hline N1 & $286(74.5)$ & $129(74.2)$ & \\
\hline N2 & $28(7.3)$ & $23(16.2)$ & \\
\hline Surgery type & & & 0.309 \\
\hline Breast-conserving surgery & $172(44.8)$ & $86(49.4)$ & \\
\hline Total mastectomy & $212(55.2)$ & $88(50.6)$ & \\
\hline Number of positive SLN(s) & & & 0.608 \\
\hline 1 & $190(49.5)$ & $94(54.0)$ & \\
\hline 2 & $144(37.5)$ & $59(33.9)$ & \\
\hline 3 & $50(13.0)$ & $21(12.1)$ & \\
\hline Residual nodal disease & & & 0.942 \\
\hline Absent & $215(56.0)$ & $98(56.3)$ & \\
\hline Present & $169(44.0)$ & $76(43.7)$ & \\
\hline Mean invasion depth in SLN (mm) & $5.3(0.3-30)$ & $5.7(0.3-23)$ & 0.294 \\
\hline Response to NAC & & & 0.128 \\
\hline Complete remission & $18(4.7)$ & $4(2.3)$ & \\
\hline Partial remission ( $\geq 30 \%$ reduction) & $269(70.1)$ & $137(78.7)$ & \\
\hline Stable disease & $90(23.4)$ & $29(16.7)$ & \\
\hline Progressive Disease & $7(1.8)$ & $4(2.3)$ & \\
\hline \multicolumn{4}{|l|}{ SLN: sentinel lymph node } \\
\hline \multicolumn{4}{|l|}{ NAC: neoadjuvant chemotherapy } \\
\hline $\begin{array}{l}\text { Response to NAC: followed the Revis } \\
\text { guideline, version } 1.1\end{array}$ & nse Evaluation C & a in Solid Tumors ( & ST) \\
\hline
\end{tabular}

\section{Factors for predicting NSLNM and the development of a nomogram}

Our goal was to develop a tool that could link routinely measured clinical factors to the actual probability of NSLNM during surgery. Table 3 displays the detailed analysis results. In the univariate analysis, the number of positive SLN(s), number of frozen nodes, tumour grade, ER and PR positivity, clinical N stage and biological subtype were found to be significantly associated with the possibility of residual nodal 
disease. The odds ratio increased in parallel with the number of positive SLN(s) and the initial N stage. In a multivariate stepwise logistic regression analysis, the number of metastatic $S L N(\mathrm{~s})$, number of frozen SLN(s), PR positivity and preoperative clinical $\mathrm{N}$ stage were found to be the independent predictors of NSLNM. These four variables were included to develop the nomogram (Fig. 1.) The Area under the receiver operating characteristics curve value of this formula was $0.709(95 \% \mathrm{Cl}, 0.658-0.761$, Hosmer-Lemeshow test p-value 0.176 ) for the development set (Table 4.) 
Table 3

Result of logistic regression for residual nodal disease

Parameter

2

3

Number of 1

positive

SLN(s)

\section{Univariable}

Multivariable

Odds $\quad 95 \% \mathrm{Cl}$
ratio

1
Odds $\quad 95 \% \mathrm{Cl}$

$\begin{array}{ll}\text { p- } & \text { Odds } \\ \text { value } & \text { ratio }\end{array}$

$<0.001 \quad 1$
$\mathrm{P}$

value

0.001

$\begin{array}{llllllllll} & 2 & 1.836 & 1.180 & 2.856 & 0.007 & 2.040 & 1.279 & 3.256 & 0.003 \\ & 3 & 2.754 & 1.453 & 5.219 & 0.002 & 3.027 & 1.571 & 5.831 & 0.001 \\ \begin{array}{l}\text { Number of } \\ \text { frozen }\end{array} & & 0.848 & 0.747 & 0.963 & 0.011 & 0.770 & 0.670 & 0.885 & 0.000 \\ \begin{array}{l}\text { SLN(s) } \\ \text { N }\end{array} & & & & & & & & & \end{array}$

Invasion

$\begin{array}{llll}1.047 & 0.995 & 1.103 & 0.079\end{array}$

depth of

SLN(s)

Age at

$\begin{array}{llll}1.005 & 0.983 & 1.028 & 0.645\end{array}$

diagnosis

Tumour

grade

1

0.040

$\begin{array}{lllll}2 & 1.491 & 0.350 & 6.348 & 0.589 \\ 3 & 0.748 & 0.164 & 3.412 & 0.708\end{array}$

HER2 status 1

$\begin{array}{llll}0.742 & 0.454 & 1.212 & 0.233\end{array}$

Oestrogen

$0-2 \quad 1$

receptor

$>2 \quad 2.241 \quad 1.301 \quad 3.860 \quad 0.004$

Progesterone $\quad 0-2$ receptor

1

$\begin{array}{lllllllll}>2 & 1.901 & 1.220 & 2.964 & 0.005 & 2.142 & 1.343 & 3.416 & 0.001\end{array}$

Biological

$\begin{array}{lllll}\text { 1: LumA } & 1.892 & 0.935 & 3.831 & 0.076\end{array}$

subtype

\begin{tabular}{lllll} 
2: LumB & 1.997 & 0.845 & 4.718 & 0.115 \\
\hline 3: HER2 & 0.615 & 0.220 & 1.723 & 0.355 \\
$\begin{array}{l}\text { 4: Triple } \\
\text { negative }\end{array}$ & 1 & & & 0.012
\end{tabular}

Ki-67 status

$\begin{array}{llll}0.995 & 0.987 & 1.003 & 0.238\end{array}$




\begin{tabular}{|c|c|c|c|c|c|c|c|c|c|}
\hline & \multirow[b]{2}{*}{$>20$} & \multicolumn{4}{|c|}{ Univariable } & \multicolumn{4}{|c|}{ Multivariable } \\
\hline & & 1.136 & 0.712 & 1.813 & 0.593 & & & & \\
\hline \multirow{4}{*}{$\begin{array}{l}\text { Initial T } \\
\text { stage }\end{array}$} & 1 & 1 & & & 0.112 & & & & \\
\hline & 2 & 1.149 & 0.538 & 2.457 & 0.719 & & & & \\
\hline & 3 & 2.016 & 0.886 & 4.585 & 0.095 & & & & \\
\hline & 4 & 1.190 & 0.308 & 4.604 & 0.801 & & & & \\
\hline \multirow{3}{*}{$\begin{array}{l}\text { Initial N } \\
\text { stage }\end{array}$} & 0 & 1 & & & $<0.001$ & 1 & & & $<0.001$ \\
\hline & 1 & 2.269 & 1.275 & 4.035 & 0.005 & 2.584 & 1.438 & 4.642 & 0.002 \\
\hline & 2 & 5.667 & 2.187 & 14.683 & 0.000 & 5.743 & 2.240 & 14.722 & 0.000 \\
\hline \multirow[t]{4}{*}{$\begin{array}{l}\text { Response to } \\
\text { NAC }\end{array}$} & $\begin{array}{l}1= \\
100 \%\end{array}$ & 1 & & & 0.958 & & & & \\
\hline & $\begin{array}{l}2=30- \\
99 \%\end{array}$ & 0.836 & 0.444 & 1.573 & 0.579 & & & & \\
\hline & $\begin{array}{l}3=0- \\
29 \%\end{array}$ & 0.851 & 0.417 & 1.738 & 0.658 & & & & \\
\hline & $4=<0 \%$ & 0.818 & 0.164 & 4.072 & 0.806 & & & & \\
\hline
\end{tabular}

Table 4

Discrimination and calibration ability of the developed nomogram

\begin{tabular}{|c|c|c|c|c|c|c|c|c|c|}
\hline & & \multirow[b]{2}{*}{$\mathrm{N}$} & \multirow[b]{2}{*}{ Residual } & \multirow[b]{2}{*}{ AUC } & & \multirow[b]{2}{*}{$\begin{array}{l}95 \% \\
\mathrm{Cl}\end{array}$} & \multicolumn{3}{|c|}{ Hosmer-Lemeshow test } \\
\hline & & & & & & & $\begin{array}{l}\text { X- } \\
\text { squared }\end{array}$ & DF & $\begin{array}{l}\mathrm{P} \\
\text { value }\end{array}$ \\
\hline Development & $\begin{array}{l}2005- \\
2016\end{array}$ & 384 & 169 & 0.709 & 0.658 & 0.761 & 8.957 & 6 & 0.176 \\
\hline Validation & $\begin{array}{l}2017- \\
2019\end{array}$ & 174 & 76 & 0.715 & 0.634 & 0.796 & 10.535 & 6 & 0.104 \\
\hline \multicolumn{10}{|c|}{ AUC $=C$ statistics } \\
\hline \multicolumn{10}{|c|}{$\begin{array}{l}\text { *Optimism corrected } \mathrm{C} \text { statistics by } 1000 \text { bootstrap } \\
\text { resamples }\end{array}$} \\
\hline
\end{tabular}

In order to test the discrimination ability of the nomogram, we conducted an independent validation study using the data from a different cohort of 174 patients who underwent surgery at the AMC from 2017 to 2019. The Area under the receiver operating characteristics curve value of this formula was $0.715(95 \% \mathrm{Cl}$, 0.634-0.796, Hosmer-Lemeshow test p-value 0.104) for the validation set (Table 4., Fig. 2.) Since the null hypothesis $\left(\mathrm{H}_{0}\right)$ for this Hosmer-Lemeshow's test was that this model was a good fit for the data, a $\mathrm{p}$ - 
value greater than 0.05 was interpreted as indicating that this prediction model was suitable for explaining the data.

Table 5 describes the sensitivity, specificity, positive predictive value and negative predictive value according to each threshold value for the assessment of the nomogram. A positive predictive value of this table indicates the ability to predict the actual probability of remnant malignant nodal disease when a surgeon proceeded to a full ALND after a positive SNB result was reported.

Table 5

Specific parameter values of the nomogram according to various cutoffs.

\begin{tabular}{|lllll|}
\hline Threshold for probability (\%) & Sensitivity (\%) & Specificity (\%) & PPV (\%) & $\begin{array}{l}\text { NPV } \\
(\%)\end{array}$ \\
\hline 15 & 99 & 4 & 45 & 80 \\
\hline 22 & 95 & 18 & 48 & 78 \\
\hline 28 & 90 & 31 & 51 & 76 \\
\hline 43 & 73 & 64 & 61 & 70 \\
\hline 63 & 25 & 89 & 64 & 54 \\
\hline 67 & 14 & 95 & 69 & 52 \\
\hline 84 & 1 & 100 & 100 & 50 \\
\hline PPV : positive predictive value & & & & \\
\hline NPV : negative predictive value & & & & \\
\hline
\end{tabular}

\section{Discussion}

Despite the fact that traditional axillary surgery remains one of the standard management options, optimal treatment of the axilla has been an evolving area aimed at reducing its related morbidity. According to the most recent NCCN guideline for invasive breast cancer, we should perform standard ALND or SLNB in selected cases when nodes are clinically negative after NAC and FNA or core biopsy is positive prior to preoperative chemotherapy [4]. ALND, as a means for achieving local disease control, carries an indisputable and often unacceptable risk of complications such as seroma, infection and lymphedema [8]. However, previous studies that analysed patients with primary breast cancer or who underwent NAC found that $40-60 \%$ of those who underwent ALND had no residual axillary disease [5-7]. In our study population, $56 \%$ of patients had no residual nodal metastasis after completion of axillary dissection, which was performed due to a positive sentinel nodal biopsy result at the time of surgery. As a result, a substantial portion of patients may have been subjected to the significant morbidity of extensive axillary surgery without receiving any clinical benefit. Thus, the goal of our study was to find a tool that allows a surgeon to be more selective in choosing a subgroup of patients who may be spared from the possible morbidity of ALND. Our nomogram is composed of four variables: number of metastatic SLN(s), number of frozen 
nodes, PR positivity and preoperative clinical $\mathrm{N}$ stage. These parameters were available before proceeding to a full ALND.

Several models have been proposed to predict the presence of NSLNM for breast cancer patients with or without NAC [6, 9-13]. One of the most widely used nomograms was developed by Van Zee et al., which included eight statistically significant variables of pathological size, LVI, method of detection, number of positive SLNs, multifocality and number of negative SLNs [13]. ER status and nuclear grade were included in the model but failed to show significant association with the likelihood of NSLNM. The overall discriminative ability of this nomogram, as measured by the ROC curve, was 0.76 for the retrospective population. The AUC value for the corresponding prospective population was 0.77 . However, this model is only applicable to patients without NAC. Patients who were treated with NAC may need another version of the nomogram to accurately predict NSLNM. Moreover, the variables, such as pathological tumour size and LVI, may not always be available in a routine frozen section pathology during surgery.

Yu et al. recently published a nomogram based on factors such as serum tumour markers CA 15-3 and CEA [14]. They demonstrated that adding CEA and CA15-3 to the nomogram significantly improved its discrimination ability when compared to the nomogram without both serum markers (AUC, $0.773(0.732-$ $0.815)$ vs. $0.727(0.682-0.771), p<0.001)$. They also included the number of positive and negative SLNs as separate factors. However, serum CEA levels may be elevated in malignancies other than colorectal cancer. In many institutions, CEA may not be a routinely measured biomarker for breast cancer patients. Furthermore, the nomogram was developed using data from patients who underwent upfront surgery with one to two positive SLN macrometastases, so its applicability to patients who received preoperative systemic therapy is limited.

Jeruss et al. proposed a model for predicting the likelihood of NSLNM(s) in patients with a positive SLN after preoperative chemotherapy [6]. They included five clinicopathologic factors: method of detection of SLN metastasis, multicentricity, initial lymph node status, pathologic tumour size and LVI. The AUC of this model was 0.85 , and the bootstrap-corrected AUC was 0.76 . Since their study population included only patients with one metastatic sentinel node who underwent NAC, the applicability of this nomogram to patients with more than one nodal disease burden may be limited. The variables of this nomogram also included pathologic tumour size and LVI, which overlapped with the previous prediction model proposed by Van Zee et al. In many institutions, both parameters may be available only in a permanent pathology report.

Ryu et al. proposed another prediction model of NSLNM for patients who received preoperative chemotherapy [12]. They created a nomogram composed of four variables: pathologic T stage, LVI, SLN metastasis size and number of positive SLN metastases. Their nomogram exhibited the AUC of 0.791 for internal validation, while the AUC for external validation cohort was 0.705 . The number of patients included in the developing cohort was 197, while the number of patients included in the external validation was 30 . To develop the nomogram, we analysed 384 patients' data and tested the formula with 174 patients' data from different time windows. Aside from the difference in the number of patients included, 
the data involved 57 patients who were SLN negative but underwent ALND, whereas our study included only patients with one to three positive SLN(s) for analysis.

The aforementioned studies and this study share the goal of accurately predicting NSLNM, which may help a surgeon be more selective in finding full-extent ALND candidates. However, de-escalation of axillary surgery to eligible patients might raise concerns about possible residual metastatic nodal disease and the associated risk of tumour recurrence in the future. Nguyen et al. discovered a significant shift in the axillary surgery trend for clinical N1 patients treated with NAC, with SLN surgery becoming more common while ALND becoming less common [15]. Although de-escalation of axillary surgery after NAC has been an increasing trend, significant prospective data regarding disease recurrence and related survival are lacking [16].

A retrospective study compared the survival result between SLNB alone and full-extent ALND in patients with one to three positive sentinel nodes on intraoperative frozen biopsy after preoperative chemotherapy [17]. After a median follow-up of 59.4 months for 483 patients (SLNB alone 188 and ALND 295, respectively), they found no significant difference in survival between the two groups of patients. Their analysis suggested that limited axillary surgery may be a possible surgical option for selected eligible patients.

On the other hand, Almahariq et al. reported that SLNB alone was associated with significantly lower survival than ALND group (HR 1.7, 95\% Cl 1.3-2.2, $\mathrm{p}<0.001$ ), with estimated 5-year overall survival of $71 \%$ in SLNB-only group compared with $77 \%$ of ALND group ( $p=0.01)$, when they compared the survival of a total of 1617 eligible ypN1 patients in the National Cancer Database [18]. However, they found that SLNB may have comparable result with ALND in the selected patients with luminal A or B tumours with a single metastatic lymph node disease (HR $1.03,95 \% \mathrm{Cl} 0.59-1.8, \mathrm{p}=0.91)$. They were cautious about reducing the extent of axillary surgery, but they also demonstrated that limiting axillary surgery may be feasible in some selected patients with favourable tumour biology. Until further conclusive clinical data is published, we believe that a more cautious approach to ypN1 breast cancer patients is still appropriate, but it is worthwhile to try to be more selective in choosing eligible patients for reduced range of axillary management.

There are several limitations to this study. Given the single institutional, retrospective nature of this study, we acknowledge the potential existence of selection bias among eligible patients. There was observed heterogeneity in baseline patient characteristics between the test group and validation group. The baseline group had a higher proportion of clinical NO patients, whereas the validation cohort had a higher proportion of clinical N2 patients. However, throughout the entire patient data analysis, we found significant differences in the baseline clinicopathologic factors between the cohort with non-sentinel node metastasis and the cohort without residual nodal disease. In addition, it is significant to show that this is the data that most accurately reflects real-world practice and demonstrates the recent trend of changes in surgical methods used by operators. Despite its different period of treatment time window, the resulting nomogram was validated only in a patient cohort from a single institution. External validation with 
sufficient number of patients and patients with different background demographic data needs to be done to further validate the correlation of this proposed nomogram.

\section{Conclusions}

The ultimate goal would be to tailor appropriate axillary surgery based on each patients' disease status, so that only patients who are expected to benefit from ALND are subjected to the possible morbidity. Moreover, we hope to spare patients who may not gain significant benefit from the extensive procedure. For patients with one to three positive SLN(s) after preoperative chemotherapy, this nomogram could provide clinically useful information to a surgeon about whether to proceed with further axillary dissection. As a result, we may have an additional guiding tool to decide ALND intraoperatively, allowing a patient to avoid a separate surgery session of ALND.

\section{Declarations}

\section{Funding}

This study was supported by the Asan Institute for Life Sciences, Asan Medical Center, Seoul, Korea (Grant Number 2020-0037).

\section{Competing Interests}

The authors declare that they have no conflict of interest.

\section{Author Contributions}

Jung Whan Chun wrote the main manuscript text. Sae Byul Lee designed and conducted the research. Jong Won Lee, Hee Jeong Kim, II Yong Chung, Jisun Kim, Beom Seok Ko, Byung Ho Son, and Sei-Hyun Ahn supervised the process of the research. All authors reviewed the manuscript.

\section{Data availability}

The datasets analysed during the current study are not publicly available due to individual privacy (which included patient verification number of the institution) but are available from the corresponding author on reasonable request.

\section{Ethical approval}

All procedures involving human participants were performed in accordance with the ethical standards of the institutional and/or national research committee and with the 1964 Helsinki Declaration and its later amendments or comparable ethical standards

\section{References}


1. King TA, Morrow M (2015) Surgical issues in patients with breast cancer receiving neoadjuvant chemotherapy. Nat Rev Clin Oncol 12(6):335-343

2. El Hage Chehade H, Headon H, El Tokhy O, Heeney J, Kasem A, Mokbel K (2016) Is sentinel lymph node biopsy a viable alternative to complete axillary dissection following neoadjuvant chemotherapy in women with node-positive breast cancer at diagnosis? An updated meta-analysis involving 3,398 patients. Am J Surg 212(5):969-981

3. Pilewskie M, Morrow M (2017) Axillary Nodal Management Following Neoadjuvant Chemotherapy: A Review. JAMA Oncol 3(4):549-555

\section{4. <NCCN guideline 2021_ver7.pdf>.}

5. Dingemans SA, de Rooij PD, van der Vuurst RM, Budel LM, Contant CM, van der Pool AE (2016) Validation of Six Nomograms for Predicting Non-sentinel Lymph Node Metastases in a Dutch Breast Cancer Population. Ann Surg Oncol 23(2):477-481

6. Jeruss JS, Newman LA, Ayers GD, Cristofanilli M, Broglio KR, Meric-Bernstam F, Yi M, Waljee JF, Ross MI, Hunt KK (2008) Factors predicting additional disease in the axilla in patients with positive sentinel lymph nodes after neoadjuvant chemotherapy. Cancer 112(12):2646-2654

7. Gimbergues P, Abrial C, Durando X, Le Bouedec G, Cachin F, Penault-Llorca F, Mouret-Reynier MA, Kwiatkowski F, Maublant J, Tchirkov A et al (2009) Clinicopathological factors and nomograms predicting nonsentinel lymph node metastases after neoadjuvant chemotherapy in breast cancer patients. Ann Surg Oncol 16(7):1946-1951

8. Giuliano AE, Hunt KK, Ballman KV, Beitsch PD, Whitworth PW, Blumencranz PW, Leitch AM, Saha S, McCall LM, Morrow M (2011) Axillary dissection vs no axillary dissection in women with invasive breast cancer and sentinel node metastasis: a randomized clinical trial. JAMA 305(6):569-575

9. Degnim AC, Reynolds C, Pantvaidya G, Zakaria S, Hoskin T, Barnes S, Roberts MV, Lucas PC, Oh K, Koker $\mathrm{M}$ et al (2005) Nonsentinel node metastasis in breast cancer patients: assessment of an existing and a new predictive nomogram. Am J Surg 190(4):543-550

10. Hwang RF, Krishnamurthy S, Hunt KK, Mirza N, Ames FC, Feig B, Kuerer HM, Singletary SE, Babiera G, Meric $F$ et al (2003) Clinicopathologic factors predicting involvement of nonsentinel axillary nodes in women with breast cancer. Ann Surg Oncol 10(3):248-254

11. Kohrt HE, Olshen RA, Bermas HR, Goodson WH, Wood DJ, Henry S, Rouse RV, Bailey L, Philben VJ, Dirbas FM et al (2008) New models and online calculator for predicting non-sentinel lymph node status in sentinel lymph node positive breast cancer patients. BMC Cancer 8:66

12. Ryu JM, Lee SK, Kim JY, Yu J, Kim SW, Lee JE, Han SH, Jung YS, Nam SJ (2017) Predictive Factors for Nonsentinel Lymph Node Metastasis in Patients With Positive Sentinel Lymph Nodes After Neoadjuvant Chemotherapy: Nomogram for Predicting Nonsentinel Lymph Node Metastasis. Clin Breast Cancer 17(7):550-558

13. Van Zee KJ, Manasseh DM, Bevilacqua JL, Boolbol SK, Fey JV, Tan LK, Borgen PI, Cody HS 3, Kattan MW (2003) A nomogram for predicting the likelihood of additional nodal metastases in breast cancer patients with a positive sentinel node biopsy. Ann Surg Oncol 10(10):1140-1151 
14. Yu Y, Wang Z, Wei Z, Yu B, Shen P, Yan Y, You W (2021) Development and validation of nomograms for predicting axillary non-SLN metastases in breast cancer patients with 1-2 positive sentinel lymph node macro-metastases: a retrospective analysis of two independent cohorts. BMC Cancer 21(1):466

15. Nguyen TT, Hoskin TL, Day CN, Degnim AC, Jakub JW, Hieken TJ, Boughey JC (2018) Decreasing Use of Axillary Dissection in Node-Positive Breast Cancer Patients Treated with Neoadjuvant Chemotherapy. Ann Surg Oncol 25(9):2596-2602

16. Nguyen TT, Boughey JC (2018) ASO Author Reflections: Rate of Axillary Lymph Node Dissection has Decreased in Patients Treated with Neoadjuvant Systemic Therapy. Ann Surg Oncol 25(Suppl 3):693694

17. Chun JW, Kim J, Chung IIY, Ko BS, Kim HJ, Lee JW, Son BH, Ahn SH, Lee SB (2021) Comparison of survival outcomes for axillary surgery extent based on intraoperative sentinel lymph node biopsy result after neoadjuvant chemotherapy for breast cancer. Breast Cancer Res Treat 187(3):647-655

18. Almahariq MF, Levitin R, Quinn TJ, Chen PY, Dekhne N, Kiran S, Desai A, Benitez P, Jawad MS, Gustafson GS et al: Omission of Axillary Lymph Node Dissection is Associated with Inferior Survival in Breast Cancer Patients with Residual N1 Nodal Disease Following Neoadjuvant Chemotherapy. Ann Surg Oncol 2021, 28(2):930-940.

\section{Figures}

Pains

meta in SNB

frowennode

PrePR

Clinical $N$

Total Points

Predcted probabily of Residual
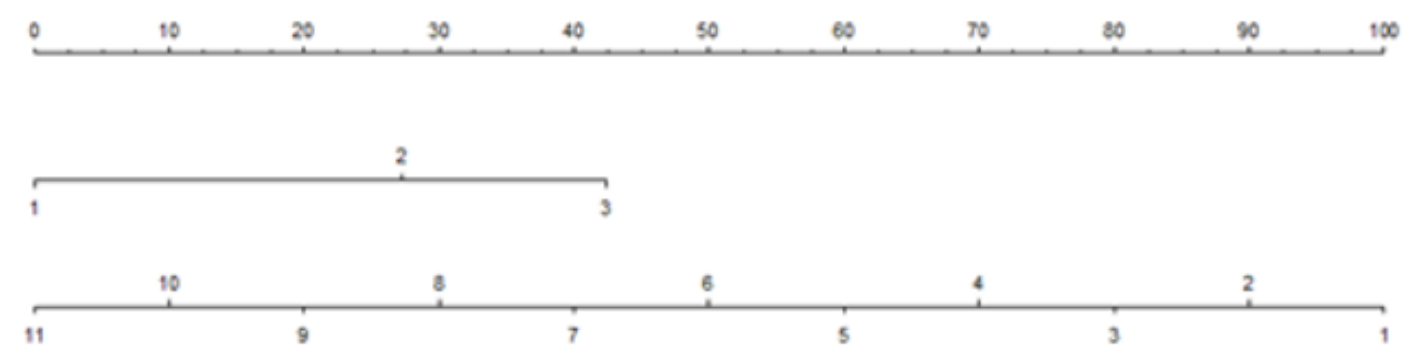

1
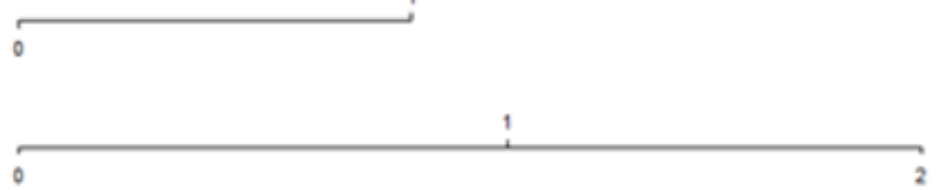

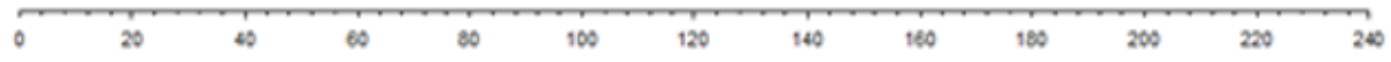

\section{Figure 1}


The nomogram to predict non-sentinel lymph node metastasis in breast cancer patients with one to three positive sentinel lymph node(s) on a frozen biopsy result

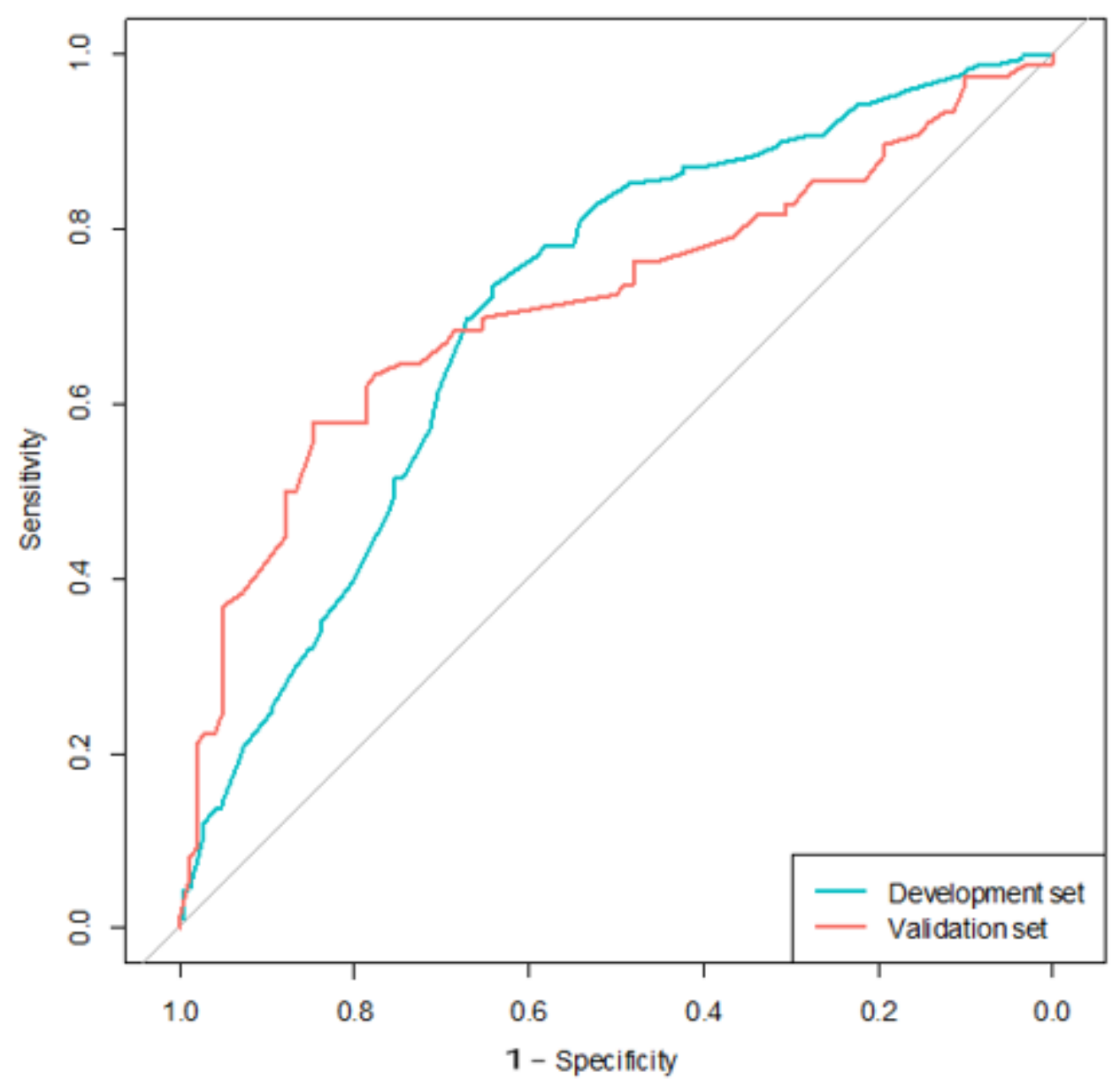

Figure 2

Receiver operating characteristics curve (ROC) of the nomogram. Area under the ROC curve was 0.709 on the development set and 0.715 on the validation set, respectively 